\title{
Optimization-Based Antenna Miniaturization Using Adaptively Adjusted Penalty Factors
}

\author{
Marzieh Mahrokh ${ }^{1, *}$ and Slawomir Koziel ${ }^{1,2}$ (D) \\ 1 Engineering Optimization and Modeling Center, Reykjavik University, 102 Reykjavik, Iceland; Koziel@ru.is \\ 2 Faculty of Electronics, Telecommunications and Informatics, Gdańsk University of Technology, \\ 80-233 Gdańsk, Poland \\ * Correspondence: marziehm@ru.is; Tel.: +354-599-6376
}

Citation: Mahrokh, M.; Koziel, S. Optimization-Based Antenna Miniaturization Using Adaptively Adjusted Penalty Factors. Electronics 2021, 10, 1751. https://doi.org/ 10.3390/electronics10151751

Academic Editors: Ivo Couckuyt, Domenico Spina and Luciano De Tommasi

Received: 29 June 2021

Accepted: 18 July 2021

Published: 21 July 2021

Publisher's Note: MDPI stays neutral with regard to jurisdictional claims in published maps and institutional affiliations.

Copyright: (C) 2021 by the authors. Licensee MDPI, Basel, Switzerland. This article is an open access article distributed under the terms and conditions of the Creative Commons Attribution (CC BY) license (https:/ / creativecommons.org/licenses/by/ $4.0 /)$.

\begin{abstract}
The continuing trend for miniaturization of electronic devices necessitates size reduction of the comprising components and circuitry. Specifically, integrated circuit-antenna modules therein require compact radiators in applications such as $5 \mathrm{G}$ communications, implantable and on-body devices, or internet of things (IoT). The conflict between the demands for compact size and electrical and field performance can be mitigated by means of constrained numerical optimization. Evaluation of performance-related constraints requires expensive electromagnetic (EM) analysis of the system at hand; therefore, their explicit handling is inconvenient. A workaround is the penalty function approach where the primary objective (typically, antenna size) is complemented by additional terms quantifying possible constraint violations. The penalty coefficients that determine contributions of these terms are normally adjusted manually, which hinders precise control over antenna performance figures and often leads to inferior results in terms of achieved miniaturization rates. This paper proposes a novel algorithm featuring an automated adjustment of the penalty factors throughout the optimization process. Our methodology is validated using three broadband antenna structures. The obtained results demonstrate that the presented adaptive adjustment permits a precise control over the constraint violations while leading to better miniaturization rates as compared to manual penalty term setup.
\end{abstract}

Keywords: antenna miniaturization; compact antennas; EM-driven design; constrained optimization; penalty functions; constraint violations

\section{Introduction}

Accommodation along with the integration requirements of antennas with the circuit parts has rendered miniaturization a necessity in applications such as wireless communications, internet of things, or portable and on-body devices [1,2]. As the majority of antenna performance figures (reflection, gain, bandwidth, radiation efficiency, radiation pattern) are linked to the physical size [3], a miniaturization task is far from trivial. Miniaturization techniques based on utilization of high-permittivity substrates [3,4], have been successful in reducing the antenna size at the expense of degrading the bandwidth. Several other techniques based on geometrical modifications of the antenna structure have been proposed, including the use of meandered traces [1,5], introduction of corrugations in the ground plane and the radiator [6,7], or incorporation of L-shaped slits [1].

Although the aforementioned techniques provide degrees of freedom to work out a compromise between the antenna size and the electromagnetic (EM) performance, they also contribute to the complexity of the antenna design process, primarily due to an increase in the number of geometry parameters. The unintuitive interrelations between the antenna dimensions and the performance figures virtually eliminate the possibility of finding an optimum design using traditional tuning techniques, especially when multiple objectives are to be taken into account. The only way to produce the optimum design is through simultaneous numerical optimization of all geometrical parameters. Depending on the 
antenna type, available initial design, and the design goals and constraints, this can be done with either global [8-10], quasi-global [11], or local search routines [12,13].

The reliability of the optimization process is strongly correlated with the accuracy of the computational model, which, most often, is based on full-wave electromagnetic (EM) analysis. Conventional optimization routines require numerous EM simulations, which entails significant computational costs. To alleviate this difficulty, a number of techniques have been proposed, including adjoint sensitivities [14-19], surrogate-based methods involving multi-fidelity simulation models [13,20-22], response surface approximations [23], several variations of space mapping (SM) [24] (e.g., aggressive space mapping [25], implicit SM [26]), feature-based optimization [27], but also machine learning methods [28,29], and surrogate-assisted versions of nature-inspired algorithms [30,31].

In the context of EM-driven antenna miniaturization, explicit reduction of the structure size (footprint area, volume) is the preferred approach because it enables direct control over the primary objective. Notwithstanding, the problem becomes a constrained task as electrical and field performance figures (impedance matching, gain, axial ratio) have to be controlled so that the appropriate levels thereof are ensured [32-34]. The principal inconvenience is that the majority of constraints are expensive to evaluate, i.e., require EM analysis of the antenna. A workaround is to handle the constraints in an implicit manner using the penalty function approach [34]. Therein, a satisfaction of the constraints is enforced by complementing the main objective with the contributions proportional to constraint violations (evaluated using suitable metrics).

The appropriate adjustment of the penalty coefficients is a non-trivial task. A coefficient that is too small, leads to underestimating the contributions of constraint violations to the objective function. Conversely, if the penalty coefficient is too large, the objective function becomes extremely steep in the vicinity of the feasible region boundary, which may cause a premature termination of the optimization process. A workaround was proposed in [35], where the acceptance threshold for maximum in-band reflection has been adaptively adjusted to facilitate exploration of the feasible space. An alternative constraint-oriented objective function has been adopted in [34] to identify the constrained optimum in the boundary of the feasible region [34], or to enable objective relaxation by switching between miniaturization and the electrical performance figures of interest [10]. However, in all cases, the efficacy of the optimization process relies on a manual selection of the penalty factors.

The discussion above indicates that the appropriate setup of the objective function, in particular, the penalty terms, is of paramount importance for the reliability and overall performance of EM-driven antenna miniaturization. This paper proposes a novel algorithm for explicit antenna size reduction, which features automated adjustment of the penalty factor values in the course of the optimization process. The adjustment process is focused on identifying the optimum penalty factor values based on current constraint violations and eliminates the need for manual, trial-and-error efforts. This, in turn, allows fulfillment of constraint satisfaction (up to the requested tolerance) while leading to improved miniaturization rates as compared to the conventional approach.

Our methodology is validated using three broadband antenna structures optimized for minimum size. Extensive benchmarking indicates that the adaptive adjustment of the penalty factors allows for a precise control over the constraint violations while leading to overall better results in terms of the achievable miniaturization rates (as compared to algorithms using fixed penalty terms).

\section{Optimization-Based Antenna Miniaturization}

This section recalls the formulation of EM-driven antenna miniaturization as a constrained numerical problem with explicit size reduction. Subsequently, we present the reference trust-region-based algorithm, as well as outline the proposed algorithm for automated adjustment of the penalty factors. 


\subsection{Problem Formulation}

We will use $\boldsymbol{R}(x)$ to denote the response of the EM simulation model of the antenna structure of interest. Here, $x$ is a vector of the geometry parameters of the structure, which are to be adjusted in the course of the optimization process. The optimization problem at hand is to minimize the antenna size $A(x)$ while ensuring acceptable levels of performance figures pertinent to electrical and field characteristics of the structure, e.g., of the standard form.

$$
s_{j}(x) \leq s_{j}, j=1, \ldots, k
$$

Note that $s_{j}(x)$ are evaluated based on the simulated antenna response $R(x)$. As mentioned before, the penalty function approach provides convenient means of handling the performance constraints, in which case the objective function can be defined as

$$
U_{A}(\boldsymbol{R}(\boldsymbol{x}))=A(\boldsymbol{x})+\beta_{1} c_{1}(\boldsymbol{x})^{2}+\ldots+\beta_{k} c_{k}(\boldsymbol{x})^{2}
$$

where $k$ is the total number of constraints, $c_{j}(x)$ is the penalty function quantifying violation of the $j$ th constraint (1), with $\beta_{j}$ being the corresponding penalty factor. The penalty functions only contribute to (2) if the corresponding constraints are violated, i.e., $s_{j}(\boldsymbol{x})>S_{j}$. A typical definition of the penalty function would be the one measuring a relative violation of the constraint, i.e.,

$$
c_{j}(\boldsymbol{x})=\max \left\{\frac{\gamma_{j}}{S_{j}}, 0\right\}
$$

where $\gamma_{j}$ is the constraint violation defined as

$$
\gamma_{j}=s_{j}(\boldsymbol{x})-s_{j}
$$

The design task is defined as a nonlinear minimization problem of the form.

$$
x^{*}=\underset{x \in X}{\operatorname{argmin}} U_{A}(\boldsymbol{R}(\boldsymbol{x}))
$$

where $X$ is the parameter space, typically determined by the lower and upper bounds for antenna geometry parameters $x$. It should be noted that without the penalty function approach, the design task would be subject to additional constraints (1). Whereas, when using (1)-(3), it becomes an unconstrained problem, apart from the aforementioned box constraints.

\subsection{Trust-Region Gradient-Based Algorithm}

The standard trust-region-based algorithm [33] is employed in this work as the core optimization engine. The procedure approximates $x^{*}$ with a series $x^{(i)}, i=0,1, \ldots$, obtained by solving

$$
\boldsymbol{x}^{(i+1)}=\arg \min _{x ;\left\|\boldsymbol{x}-\boldsymbol{x}^{(i)}\right\| \leq \delta} U_{A}\left(\boldsymbol{L}^{(i)}(\boldsymbol{x})\right)
$$

In (6), $L^{(i)}(x)$ is a linear (first-order Taylor) approximation of $R\left(x^{(i)}\right)$. The candidate solution rendered by (6) is only accepted if $U_{A}\left(\boldsymbol{R}\left(x^{(i+1)}\right)\right)<U_{A}\left(\boldsymbol{R}\left(x^{(i)}\right)\right)$. The trust region radius $\delta$ is adaptively adjusted based on a gain ratio calculated as

$$
\rho_{A}=\frac{U_{A}\left(\boldsymbol{R}\left(\boldsymbol{x}^{(i+1)}\right)\right)-U_{A}\left(\boldsymbol{R}\left(\boldsymbol{x}^{(i)}\right)\right)}{U_{A}\left(\boldsymbol{L}^{(i)}\left(\boldsymbol{x}^{(i+1)}\right)\right)-U_{A}\left(\boldsymbol{L}^{(i)}\left(\boldsymbol{x}^{(i)}\right)\right)}
$$

The algorithm is terminated when the trust region radius is diminished below a certain user-defined limit $\delta_{1}$, or convergence in the argument is achieved, i.e., ||$x^{(i+1)}-x^{(i)}|| \leq \delta_{x}$. 


\section{Size Reduction with Adaptive Penalty Coefficients}

Formulation (1) of the objective function offers an efficient way of handling the design constraints, especially those that are expensive to evaluate (i.e., require EM analysis of the antenna). Notwithstanding, an appropriate setup of penalty coefficients is a non-trivial matter and may affect the performance of the optimization process as elaborated on in Section 1.

This work proposes a novel algorithm incorporating adaptive adjustment of penalty coefficients in the course of the optimization run. The presented procedure eliminates the need for trial-and-error objective function setup as well as leads to improved antenna miniaturization rates, as demonstrated in Section 4 . In the following, we discuss the penalty factor adjustment concept (Section 3.1), the adjustment procedure (Section 3.2), as well as the complete optimization algorithm (Section 3.3).

This section recalls the formulation of EM-driven antenna miniaturization as a constrained numerical.

\subsection{Adaptively Adjusted Penalty Factors. The Concept}

As mentioned before, an appropriate setup of penalty coefficients is of utmost importance for the performance of the optimization process. On the one hand, having the coefficients set at too low values will result in excessive violations of the constraints. On the other hand, the values that are too high make the optimization problem numerically challenging, especially in the context of size reduction, which requires exploration of the feasible region boundary.

The objective of the technique presented in this is to automate the process of setting up the penalty coefficients so that their specific values are based on currently detected constraint violations. We use the following prerequisites, which involve the current constraint violations as well as their possible improvements over the last consecutive iterations:

- If the parameter vector $x^{(i+1)}$ produced at the iteration $i$ is feasible from the point of view of the $j$ th constraint, the corresponding penalty coefficient $\beta_{j}$ may be reduced;

- If $x^{(i+1)}$ is infeasible but the violation of the $j$ th constraint was reduced to a sufficient extent w.r.t. the $(i-1)$ th iteration, the coefficient $\beta_{j}$ remains intact;

- If $x^{(i+1)}$ is infeasible and there was no improvement of the $j$ th constraint violation or the improvement was insufficient, the coefficient $\beta_{j}$ should be increased.

The above can be viewed as a set of rules (applied to each and every constraint), which are simple, yet allow us to relax the 'pressure' from the penalty terms when the algorithm operates in a feasible region and increase it if the discussed indicators show the lack of improvement in terms of reducing constraint violations. Furthermore, implementing these rules facilitates exploration of the feasible region boundary, which is where the minimum-size design is likely to reside. The notion of the aforementioned sufficient constraint violation improvement will be specified and discussed at length below, along with providing a rigorous formulation of the adjustment rules. To clarify the matter, Figure 1, conceptually illustrates the possible situations and the actions performed. 


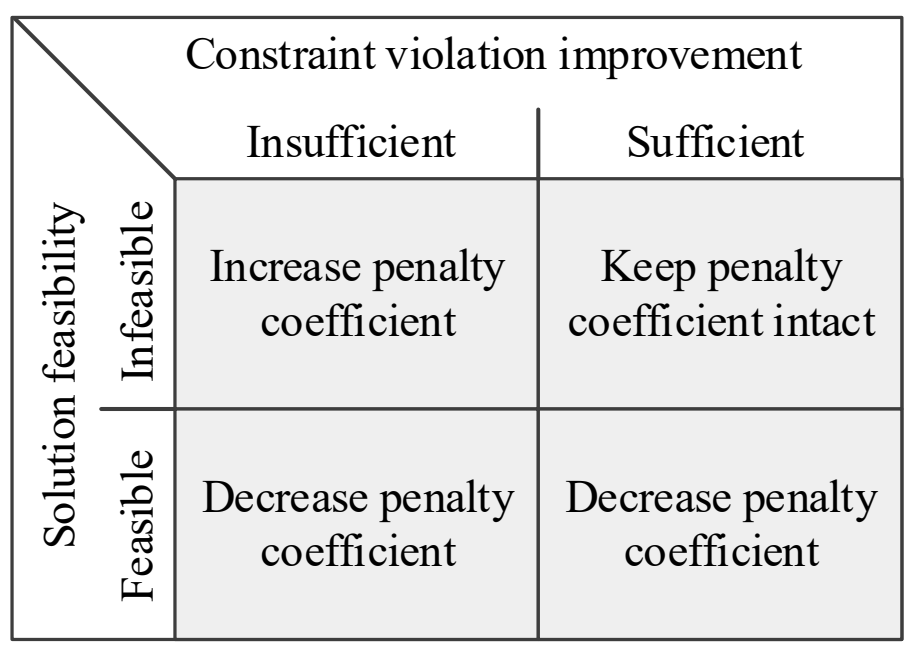

Figure 1. The concept of adaptive adjustment of penalty coefficients. Shown are the four possible situations concerning constraint violation, and the actions undertaken.

\subsection{Adaptively Adjusted Penalty Factors. The Procedure}

In order to formalize the set of penalty factor adjustment rules considered in Section 3.1, we need to quantify the sufficient constraint violation improvement, which will be defined, for the $j$ th constraint, as

$$
\Delta_{j}=M \gamma_{j}
$$

In (8), $\gamma_{j}$ is the absolute violation, cf. (4), whereas $0<M<1$ is the improvement factor. We will elaborate on the selection of the value of $M$ later in this section. Both the violation and its improvement are considered in relation to the new parameter vector $x^{(i+1)}$ produced in the $i$ th iteration and the previous point $x^{(i)}$, therefore, we will use the superscript $i+1$ to specify which iteration the above quantities are referring to. In particular, we have $\gamma_{j}^{i+1}$ and $\Delta_{j}^{i+1}$ as the constraint violation at $x^{(i+1)}$ and the improvement from $x^{(i)}$ to $x^{(i+1)}$. Similarly, the penalty coefficient for the $j$ th constraint at iteration $i$ will be denoted as $\beta_{j}{ }^{i}$.

We are now in a position to formulate the adjustment rules in a rigorous manner. These are described using the following pseudocode:

$$
\begin{aligned}
& \text { if } \gamma_{j}^{i+1} \leq 0 \\
& \text { else }^{\beta_{j}{ }^{i+1}=\beta_{j}{ }^{i} / m_{\text {decr }} ;} \\
& \text { if } \gamma_{j}{ }^{i}-\gamma_{j}^{i+1}>\Delta_{j}^{i+1} \\
& \text { else } \\
& \beta_{j}{ }^{i+1}=\beta_{j}{ }^{i+1} \text {, } \\
& \text { end } \\
& \beta_{j}{ }^{i+1}=\beta_{j}{ }^{i} m_{\text {incr }} ; \\
& \text { end }
\end{aligned}
$$

The multiplication factors $m_{\text {decr }}$ and $m_{\text {incr }}$ determine the amount of penalty factor modifications. In our numerical experiments, they are set to $m_{\text {decr }}=1.25$ and $m_{\text {incr }}=5$, but their values are not critical. As discussed in Section 3.1, maintaining fixed penalty coefficients under sufficient constraint violation improvement (while the design is still infeasible) allows us to maintain stability of the optimization process, i.e., the values of $\beta_{j}$ do not bounce back and forth (i.e., are immediately increased or decreased after the design crosses the feasible region boundary). This facilitates exploration of the boundary and leads to improved size reduction ratios.

Let us now consider the improvement factor $M$ introduced in (8). Assuming that the parameter vector $x^{(i)}$ resides in the infeasible region, and sufficient constraint violation 
improvement is observed for a few consecutive iterations, say, from $i$ to $i+k$, the upper bound on the constraint violation at the iteration $i+k$ can be calculated as

$$
\gamma_{j}^{i+k} \leq M \gamma_{j}^{i+k-1} \leq M^{2} \gamma_{j}^{i+k-2} \leq \ldots \leq M^{k} \gamma_{j}^{i}
$$

As $M<1$, the constraint violation is reduced at a geometric rate, and the improvement is faster when $M$ is closer to zero. At the same time, satisfaction of the sufficient improvement condition is more demanding for lower values of $M$. For $M=0.5$, as selected in this work, we get a balance between the rate of approaching the feasible region boundary (e.g., the constraint violation is reduced to only about six percent of its original values after only four iterations), and the difficulty of satisfying the improvement condition (reduction of only half of the current violation has to be achieved per iteration).

\subsection{Optimization Framework}

This section provides a summary of the operation of the trust-region gradient-based algorithm incorporating the adaptive adjustment of penalty coefficients as presented in Section 3.2. Here is a summary of the control parameters of the algorithm:

- $\delta_{x}, \delta_{T R}$-termination thresholds (cf. Section 2.1);

- $\quad m_{\text {inc }}, m_{d e c}$-increase and decrease factors for the automated adjustment of penalty coefficients (cf. Section 3.2);

- $\quad M-a$ factor used to determine sufficient constraint violation improvement (cf. Section 3.2);

- $\quad \beta_{j}{ }^{\max }, \beta_{j}{ }^{\min }$-maximum and minimum values of penalty coefficients; $j=1, \ldots, k$;

- $\quad \beta_{j}{ }^{0}$-initial values of the penalty coefficients; $j=1, \ldots, k$;

The algorithm operation has been presented in Figure 2. Additional clarification is provided in the form of a flow diagram in Figure 3. In the algorithm, Steps 1 and 2 are used to initialize the optimization procedure. In Steps 3 and 4, the antenna response and its sensitivity matrix are evaluated using EM analysis. The linear approximation model $\boldsymbol{L}^{(i)}(\boldsymbol{x})$ of the antenna responses is constructed in Step 5, along with its corresponding objective function $U_{A}\left(L^{(i)}(x)\right.$ ) (Step 6). The candidate design is produced in Step 7 by minimizing $U_{A}\left(L^{(i)}(x)\right)$. It is validated in Steps 8 and 9, where the gain ratio is calculated for the purpose of either accepting or rejecting $x^{(i+1)}$ and computing constraint violation improvements (Step 10). The latter is then used in Step 11 to update the penalty coefficients. The termination condition for the procedure is convergence in argument or reducing the trust region size beyond the user-defined threshold (Step 12). 
1. Set the iteration counter $i=0$; provide $\boldsymbol{x}^{(i)}$;

2. Set $m_{i n c}, m_{d e c}, \beta_{j}^{i}, S_{j}, \beta_{j}^{\max }, \beta_{j}^{m i n}, j=1, \ldots, k$;

3. Evaluate antenna response $\boldsymbol{R}\left(\boldsymbol{x}^{(i)}\right)$;

4. Evaluate antenna sensitivities $J\left(x^{(i)}\right)$ using finite differentiation;

5. Construct a linear model $L^{(i)}(x)=R\left(x^{(i)}\right)+J\left(x^{(i)}\right)$. $\left(x-x^{(i)}\right)$;

6. Construct objective function $U_{A}\left(\boldsymbol{L}^{(i)}(\boldsymbol{x})\right)=A(\boldsymbol{x})+\beta_{1}{ }^{i} \cdot C_{L, 1}(\boldsymbol{x})^{2}+\ldots+\beta_{k}{ }^{i} \cdot C_{L, k}(\boldsymbol{x})^{2}$;

7. Solve $\boldsymbol{x}^{(i+1)}=\arg \min _{\boldsymbol{x} ;\left\|\boldsymbol{x}-\boldsymbol{x}^{(i)}\right\| \leq \delta} U_{A}\left(\boldsymbol{L}^{(i)}(\boldsymbol{x})\right)$;

8. Evaluate antenna response $R\left(x^{(i)}\right)$;

9. Compute gain ratio $\rho_{A}$ and update TR size $\rho_{A}=\frac{U_{A}\left(\boldsymbol{R}\left(\boldsymbol{x}^{(i+1)}\right)\right)-U_{A}\left(\boldsymbol{R}\left(\boldsymbol{x}^{(i)}\right)\right)}{U_{A}\left(\boldsymbol{L}^{(i)}\left(\boldsymbol{x}^{(i+1)}\right)\right)-U_{A}\left(\boldsymbol{L}^{(i)}\left(\boldsymbol{x}^{(i)}\right)\right)}$; if $\rho_{A}<0$,

Go to 7;

else

$$
\Delta_{j}^{i+1}=M\left(S_{j}\left(\boldsymbol{x}^{(i)}\right)-S_{j}\right) ;
$$

end

10. if $\gamma^{i}-\gamma^{i+1}>\Delta_{j}^{i+1} \& \& \gamma^{i+1}>0$

$\beta_{i}^{i+2}=\beta_{i}^{i+1}$ mincr $_{i} i=i+1$;

elseif $\gamma^{i+1} \leq 0$

$\beta_{i}^{i+1}=\beta_{i}^{i} / m_{\text {decr }} ; i=i+1 ;$

else

$$
\beta_{i}^{i+1}=\beta_{j}^{i} ; i=i+1 ;
$$

end

11. if ||$x^{(i+1)}-x^{(i)}|| \leq \delta$ OR TR $\leq \delta_{T R}$

END;

else

Go to 4;

end

Figure 2. The concept of adaptive adjustment of penalty coefficients. Shown are the four possible situations concerning constraint violation, and the actions undertaken. 


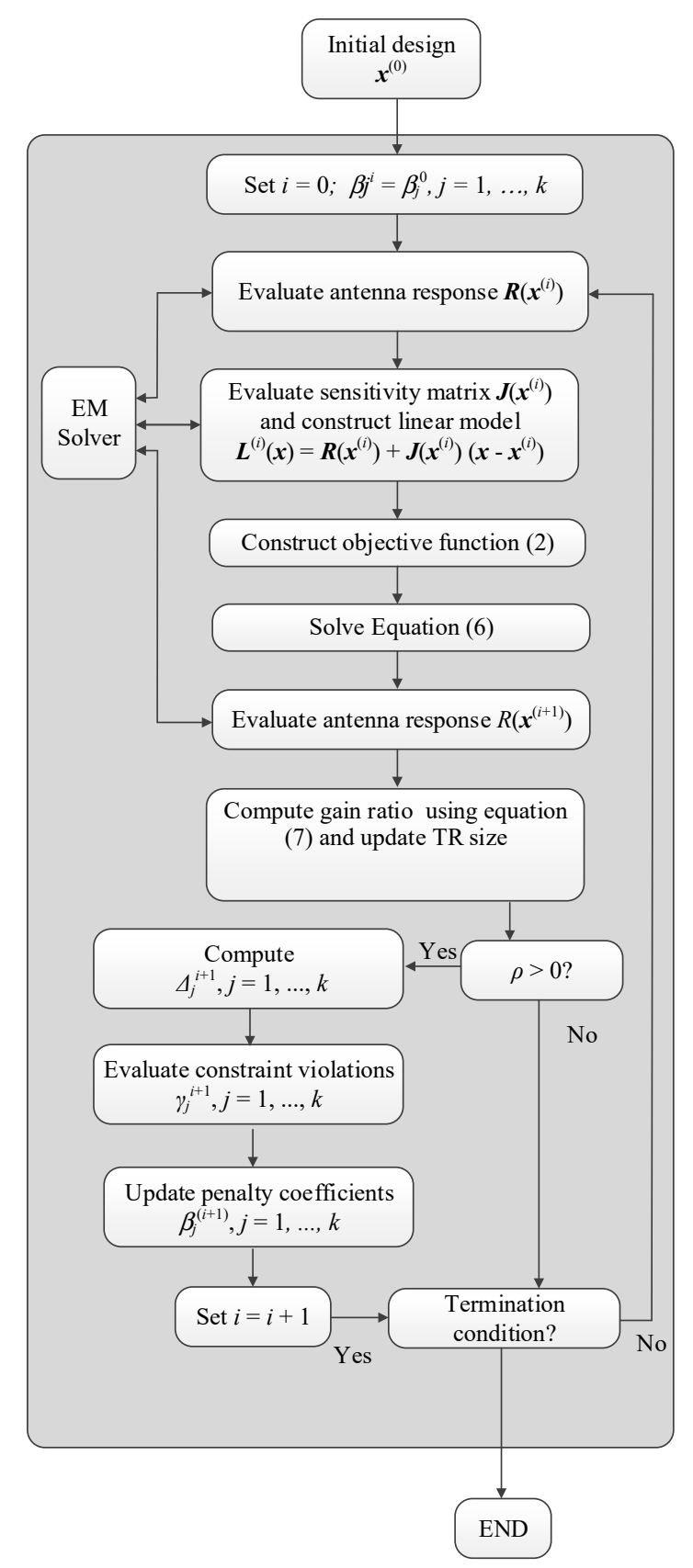

Figure 3. Operational flow of the proposed size-reduction algorithm with automated adjustment of penalty factors.

\section{Verification Case Studies}

This section provides the results of numerical experiments conducted to validate the proposed procedure for adaptive adjustment of penalty coefficients, introduced in Section 3. The verification studies involve three broadband antennas optimized for minimum size with the maximum acceptable in-band reflection considered as the only constraint. The results are compared with those obtained for manually set penalty terms, ranging from the relaxed to very tight constraint satisfaction conditions. All of the considered antenna structures have been previously described in the literature and experimentally validated therein [36-38]. Consequently, no experimental results are provided here.

The remaining part of this section is organized as follows. The experimental setup is described in Section 4.1, whereas the benchmark antennas are described along with 
the numerical results in Section 4.2, Section 4.3, and Section 4.4, respectively. Section 4.5 provides a detailed discussion.

\subsection{Experimental Setup}

The objective is to optimize the considered antennas for minimum size, as determined by the substrate area $A(x)$. The optimization process is subject to a single constraint imposed on the antenna reflection coefficient $\left|S_{11}(x)\right|$.

More specifically, we have $s_{1}(x) \leq-10$, where $s_{1}(x)$ stands for the maximum value of $\left|S_{11}(x)\right|$ within the operating frequency range of the antennas, here, $3.1 \mathrm{GHz}$ to $10.6 \mathrm{GHz}$. Correspondingly, a single penalty coefficient is used, denoted as $\beta_{1}$. The penalty function $c$ is defined based on a relative violation of the constraint, cf. (3).

The performance of the algorithm is evaluated statistically through multiple runs initiated from random starting points. This allows for reducing a possible bias associated with a particular choice of the initial design. The performance figures include the antenna size averaged over ten independent runs, and the average value of constraint violation, both evaluated at the final design yielded by the optimization algorithm.

The proposed algorithm is compared to the standard TR algorithm executed for different (fixed) values of the penalty coefficients $\beta=10^{q}, q=2,3,4,5,6$. The values set for the control parameters are $\beta_{j}{ }^{\text {min }}=100, \beta_{j}{ }^{\max }=10^{6}$, and $\beta_{j}{ }^{0}=100$. Other parameters are set as in Section 3.2. The termination thresholds are set to $\delta_{x}=\delta_{T R}=10^{-3}$.

\subsection{Antenna I}

Figure 4 shows the geometry of the first benchmark structure (Antenna I) [36]. It is a broadband monopole antenna operating within the UWB band ( $3.1 \mathrm{GHz}$ to $10.6 \mathrm{GHz}$ ).

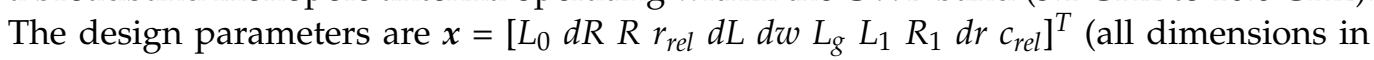
$\mathrm{mm}$ ). The feed line width is fixed to $w_{0}=1.7 \mathrm{~mm}$. The antenna is implemented on RF-35 substrate with relative permittivity $\varepsilon_{r}=3.5$, and thickness $h=0.762 \mathrm{~mm}$. The computational model incorporating the SMA connector is simulated in the time-domain solver of CST Microwave Studio.

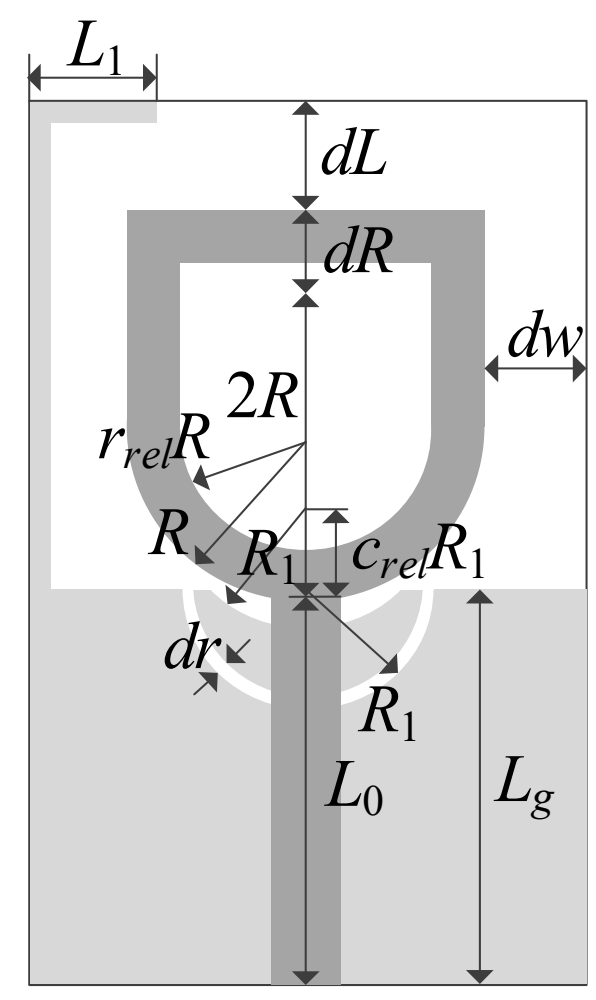

Figure 4. Geometry of Antenna I. The light gray shade is the ground plane on the backside of the structure. 
Table 1 provides the data including the average footprint area, average constraint violation, as well as the standard deviations thereof; the latter is used to quantify the repeatability of solutions. Figure 5a illustrates the reflection coefficient of Antenna I at the initial and final designs obtained for a selected run of the proposed optimization algorithm, whereas Figure $5 \mathrm{~b}$ shows the evolution of the penalty factor throughout the optimization process.

Table 1. Optimization results for antenna I.

\begin{tabular}{|c|c|c|c|c|c|c|}
\hline Performance Figure & $\begin{array}{l}\beta=10^{2} \\
\text { (Fixed) }\end{array}$ & $\begin{array}{l}\beta=10^{3} \\
\text { (Fixed) }\end{array}$ & $\begin{array}{l}\beta=10^{4} \\
\text { (Fixed) }\end{array}$ & $\begin{array}{l}\beta=10^{5} \\
\text { (Fixed) }\end{array}$ & $\begin{array}{l}\beta=10^{6} \\
\text { (Fixed) }\end{array}$ & $\begin{array}{l}\text { Adaptive } \beta \\
\text { (This Work) }\end{array}$ \\
\hline Antenna area $\left[\mathrm{mm}^{2}\right]^{1}$ & 113.7 & 250.4 & 318.6 & 331.6 & 367.6 & 222.6 \\
\hline $\operatorname{Std}(A)^{2}$ & 9.07 & 24.0 & 60.0 & 63.4 & 51.9 & 49.6 \\
\hline Constraint violation $\gamma[\mathrm{dB}]^{3}$ & 8.4 & 1.2 & 0.14 & 0.10 & 0.05 & 0.08 \\
\hline $\operatorname{Std}(\gamma)^{4}$ & 0.53 & 0.5 & 0.1 & 0.14 & 0.11 & 0.06 \\
\hline
\end{tabular}

${ }^{1}$ Average miniaturized antenna area for ten algorithm runs. ${ }^{2}$ Standard deviation of the miniaturized antenna area for ten algorithm runs.

${ }^{3}$ Average constraint violation for ten algorithm runs. ${ }^{4}$ Standard deviation of the constraint violation $\gamma$, for ten algorithm runs.

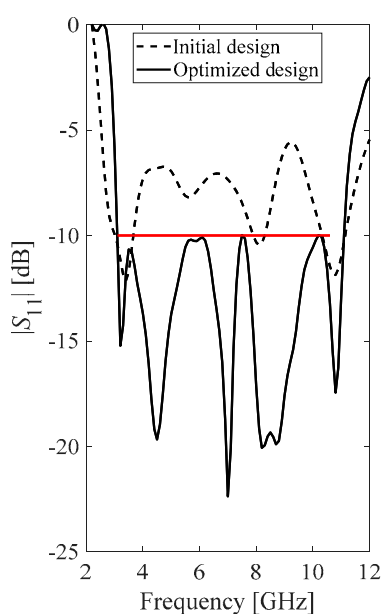

(a)

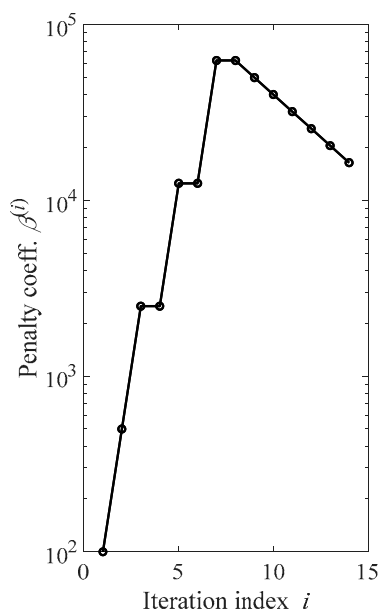

(b)

Figure 5. Antenna I: representative run of the proposed algorithm with automated adjustment of the penalty factors: (a) reflection coefficient at the initial (- - ) and optimized design (-); (b) evolution of the penalty coefficient throughout the algorithm iterations.

\subsection{Antenna II}

The second verification case is a broadband rectangular-slot monopole antenna (Antenna II), shown in Figure 6 [37], operating in the UWB range. The design parameters of the antenna are $x=\left[L_{g} L_{0} L_{s} W_{s} d d L d_{s} d W_{s} d W a b\right]^{T}$ (all in $\mathrm{mm}$ ). The feed line width is $W_{0}=3 \mathrm{~mm}$. The structure is fabricated on FR4 substrate $\left(\varepsilon_{r}=4.3, h=1.55 \mathrm{~mm}\right)$. The computational model incorporates the SMA connector, and it is simulated using the time-domain solver of CST Microwave Studio. Table 2 gathers the numerical results. Figure 7 shows the antenna responses and evolution of the penalty factor for a representative run of the proposed algorithm. 


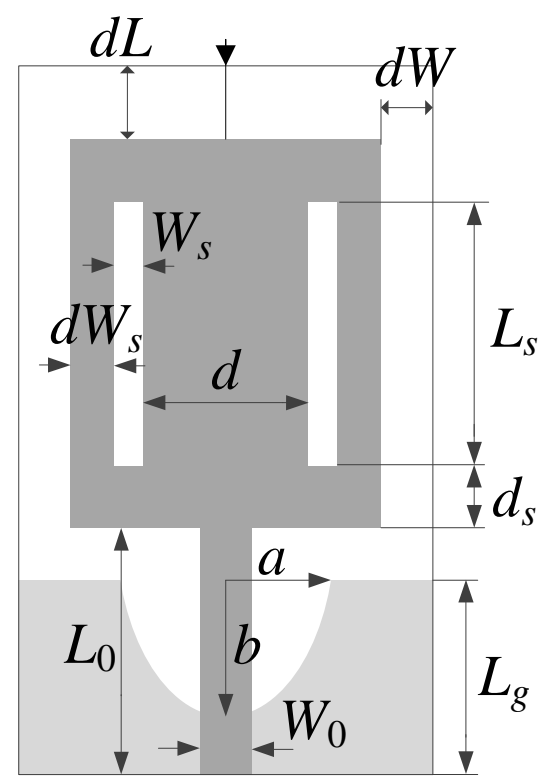

Figure 6. Geometry of Antenna II: the light gray shade is the ground plane on the backside of the structure.

Table 2. Optimization results for antenna II.

\begin{tabular}{ccccccc}
\hline Performance Figure & $\begin{array}{c}\boldsymbol{\beta}=\mathbf{1 0}^{\mathbf{2}} \\
\text { (Fixed) }\end{array}$ & $\begin{array}{c}\boldsymbol{\beta}=\mathbf{1 0}^{\mathbf{3}} \\
\text { (Fixed) }\end{array}$ & $\begin{array}{c}\boldsymbol{\beta}=\mathbf{1 0}^{\mathbf{4}} \\
\text { (Fixed) }\end{array}$ & $\begin{array}{c}\boldsymbol{\beta}=\mathbf{1 0}^{\mathbf{5}} \\
\text { (Fixed) }\end{array}$ & $\begin{array}{c}\boldsymbol{\beta}=\mathbf{1 0}^{\mathbf{6}} \\
\text { (Fixed) }\end{array}$ & $\begin{array}{c}\text { Adaptive } \boldsymbol{\beta} \\
\text { (This Work) }\end{array}$ \\
\hline Antenna area $\left[\mathrm{mm}^{2}\right]^{1}$ & 56.1 & 212.8 & 225.0 & 280.1 & 258.8 & 180.7 \\
Std $(A)^{2}$ & 3.8 & 14.3 & 25.1 & 47.4 & 29.6 & 11.1 \\
Constraint violation $\gamma[\mathrm{dB}]^{3}$ & 8.6 & 1.0 & 0.15 & 0.05 & 0.00 & 0.17 \\
$\operatorname{Std}(\gamma){ }^{4}$ & 0.60 & 0.4 & 0.10 & 0.07 & 0.01 & 0.23 \\
\hline
\end{tabular}

${ }^{1}$ Average miniaturized antenna area for ten algorithm runs. ${ }^{2}$ Standard deviation of the miniaturized antenna area for ten algorithm runs.

${ }^{3}$ Average constraint violation for ten algorithm runs. ${ }^{4}$ Standard deviation of the constraint violation $\gamma$, for ten algorithm runs.

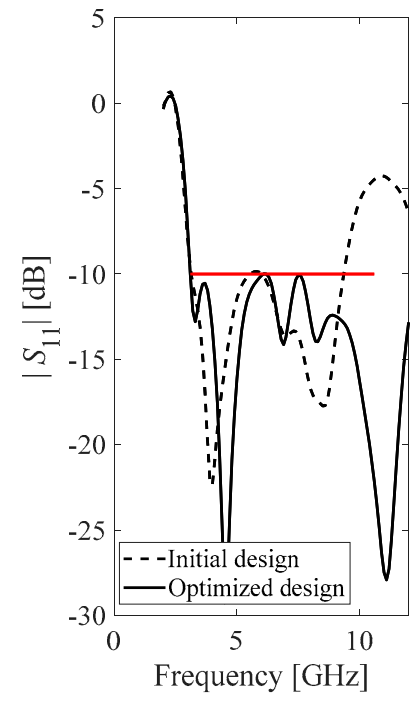

(a)

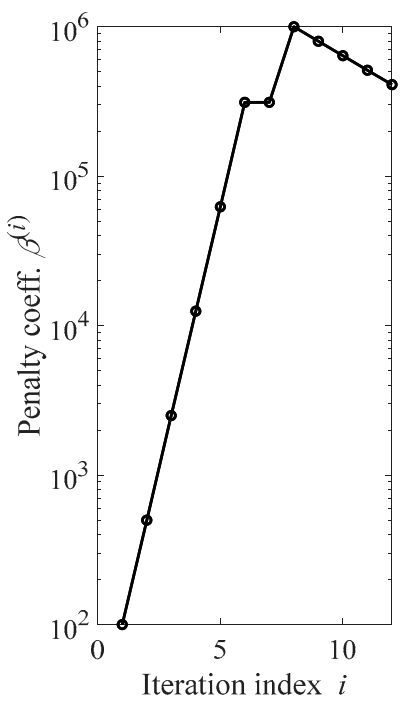

(b)

Figure 7. Antenna II: representative run of the proposed algorithm with automated adjustment of the penalty factors: (a) reflection coefficient at the initial (- - ) and optimized design (-); (b) evolution of the penalty coefficient throughout the algorithm iterations. 


\subsection{Antenna III}

Figure 8 shows Antenna III, the third verification structure. It is also a UWB monopole

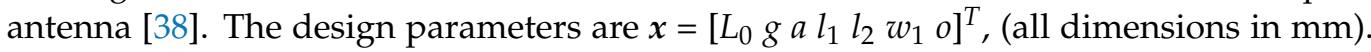
We also have $w_{0}=2 o+a$, and $w_{\mathrm{r}}=1.7 \mathrm{~mm}$. The antenna is implemented on FR4 substrate $\left(\varepsilon_{r}=4.3, h=7.62 \mathrm{~mm}\right)$. The computational model incorporates the SMA connector, and it is simulated in the time-domain solver of CST Microwave Studio.

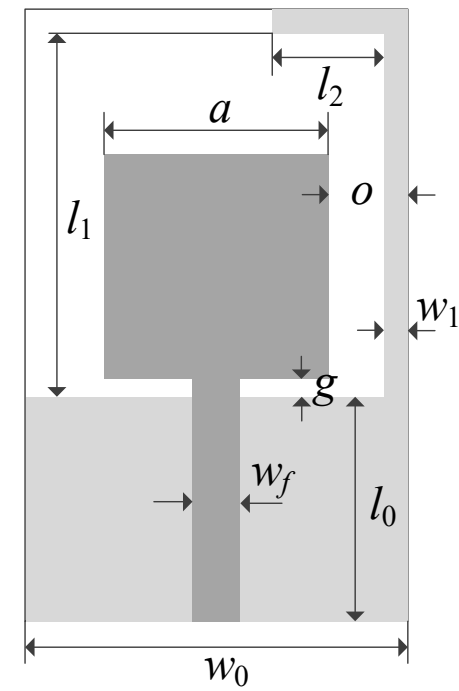

Figure 8. Geometry of Antenna III: the light gray shade is the ground plane on the backside of the structure.

Table 3 shows the numerical results, arranged the same way as for Antennas I and II. Antenna responses and evolution of the penalty coefficient for a selected algorithm run have been shown in Figure 9.

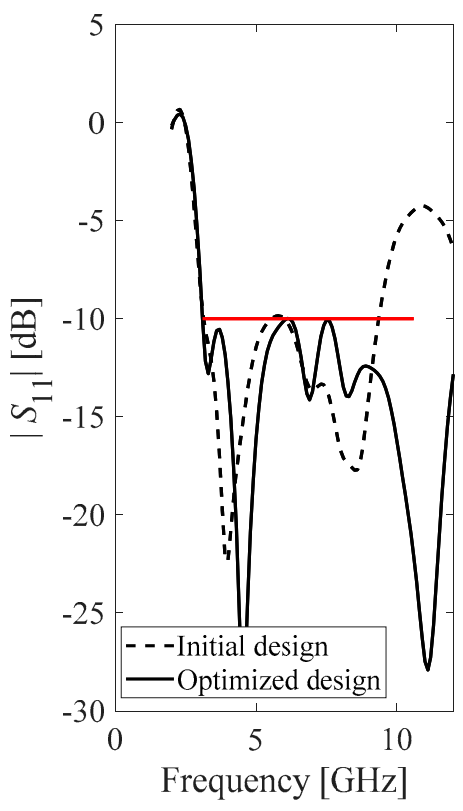

(a)

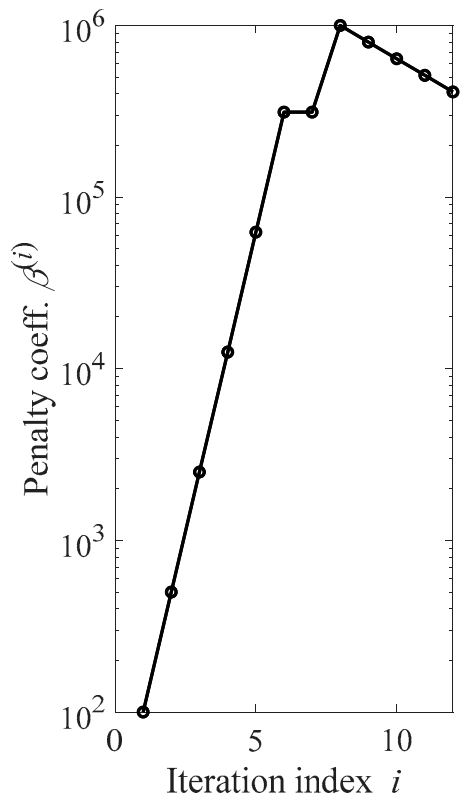

(b)

Figure 9. Antenna III: representative run of the proposed algorithm with automated adjustment of the penalty factors: (a) reflection coefficient at the initial (- - -) and optimized design (-); (b) evolution of the penalty coefficient throughout the algorithm iterations. 
Table 3. Optimization results for antenna III.

\begin{tabular}{ccccccc}
\hline Performance Figure & $\begin{array}{c}\boldsymbol{\beta}=\mathbf{1 0}^{\mathbf{2}} \\
\text { (Fixed) }\end{array}$ & $\begin{array}{c}\boldsymbol{\beta}=\mathbf{1 0}^{\mathbf{3}} \\
\text { (Fixed) }\end{array}$ & $\begin{array}{c}\boldsymbol{\beta}=\mathbf{1 0}^{\mathbf{4}} \\
\text { (Fixed) }\end{array}$ & $\begin{array}{c}\boldsymbol{\beta}=\mathbf{1 0}^{\mathbf{5}} \\
\text { (Fixed) }\end{array}$ & $\begin{array}{c}\boldsymbol{\beta}=\mathbf{1 0}^{\mathbf{6}} \\
\text { (Fixed) }\end{array}$ & $\begin{array}{c}\text { Adaptive } \boldsymbol{\beta} \\
\text { (This Work) }\end{array}$ \\
\hline Antenna area $\left[\mathrm{mm}^{2}\right]^{1}$ & 305.4 & 318.1 & 317.7 & 318.8 & 320.9 & 304.43 \\
$\operatorname{Std}(A)^{2}$ & 49.7 & 42.6 & 42.3 & 43.3 & 45.8 & 37.2 \\
${\text { Constraint violation } \gamma[\mathrm{dB}]^{3}}_{\operatorname{Std}(\gamma)^{4}}^{6.7}$ & 1.2 & 0.4 & 0.05 & 0.06 & 0.45 \\
\hline
\end{tabular}

${ }^{1}$ Average miniaturized antenna area for ten algorithm runs. ${ }^{2}$ Standard deviation of the miniaturized antenna area for ten algorithm runs.

${ }^{3}$ Average constraint violation for ten algorithm runs. ${ }^{4}$ Standard deviation of the constraint violation $\gamma$, for ten algorithm runs.

\subsection{Discussion}

The analysis of results provided in Section 4 allows for several conclusions regarding the importance of the automated adjustment of the penalty factors, as well as the performance-wise advantages of the proposed adaptive algorithm over the fixed-setup approach. To facilitate the interpretation, a graphical illustration of the data from Tables 1-3 has been provided in Figure 10. Therein, the average antenna footprint area along with the average constraint violation is shown versus the penalty coefficient for fixed-setup optimization runs. The horizontal lines represent the antenna area and constraint violation obtained for the automated adjustment procedure. The range of these lines is representative of the span in which the penalty coefficient varies throughout the optimization iterations. As it can be seen, not only the optimum value of the penalty coefficient, but also the span is problem-dependent and may not even reach the maximum set value in some cases. The following observations can be formulated:

- Although the optimum value of penalty coefficient in the fixed-setup optimization seems to be about $\beta=10^{4}$ for Antenna I, between $\beta=10^{3}$ and $\beta=10^{4}$ for Antenna II, and between $\beta=10^{4}$ and $\beta=10^{5}$ for Antenna III, considering the achievable miniaturization rates along with sufficient constraint satisfaction, the optimum value of penalty coefficient is problem-dependent. The optimum values should be identified for particular iterations of the optimization process and they are generally dependent on the status of constraint violation.

- In both fixed and automated adjustment setups, using a penalty coefficient lower than the optimum value, results in significant constraint violation. As for the former, the violation can easily become as high as five times of the tolerance threshold or even more. Antennas I and II are representative examples of this design quality degradation.

- Automated adjustment of penalty coefficients seeks to improve the final design quality by the optimum value of penalty coefficients at the level of iterations of the optimization process. This, in turn, permits a better control of constraint violations along with better achievable miniaturization rates.

- The performance improvements are significant. For the corresponding levels of constraint violations, the procedure proposed in this work leads to antenna footprints that are smaller by 110,44 , and $13 \mathrm{~mm}^{2}$ for Antenna I, II, and III, respectively. 

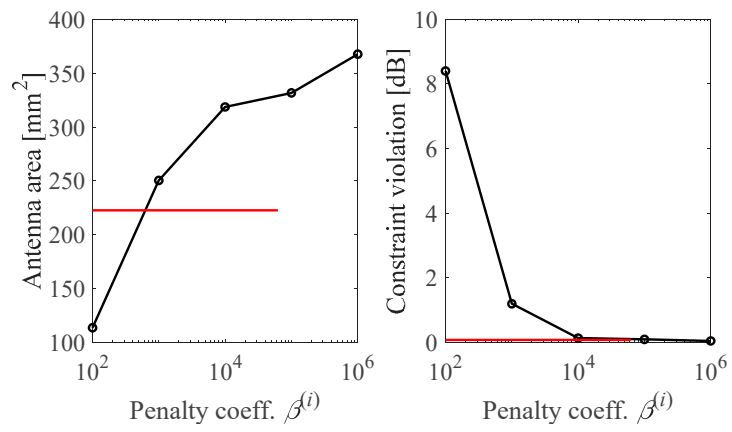

(a)
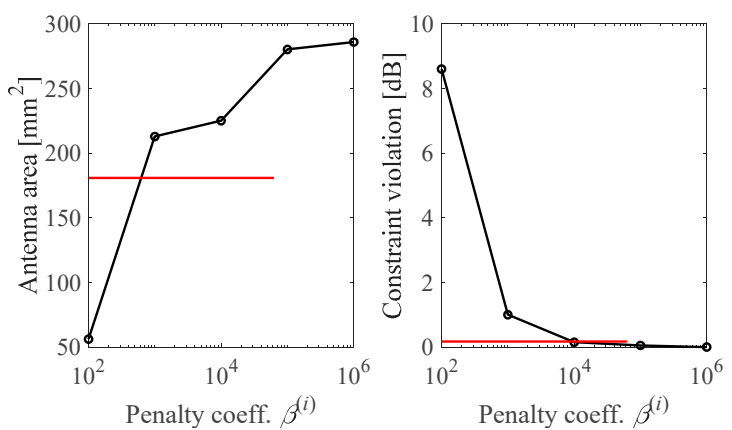

(b)
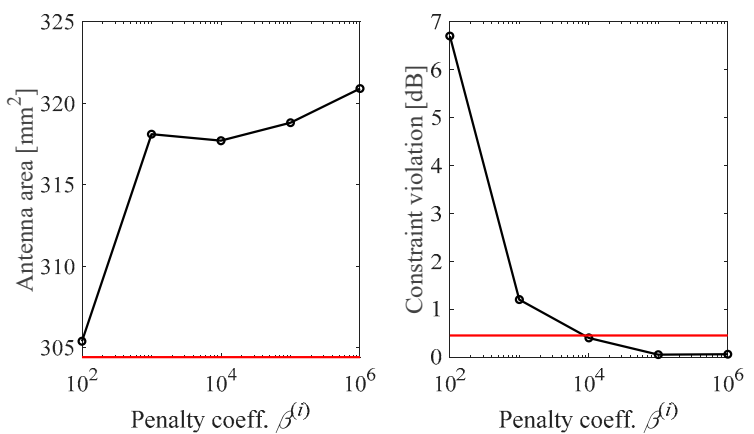

(c)

Figure 10. The average antenna footprint (left panel), and the average constraint violations (right panel) versus discrete values of the penalty coefficient utilized by the fixed-setup algorithm. The horizontal lines on the left show the average antenna footprint, whereas the ones on the right denote the average constraint violation for the designs obtained using the proposed automated adjustment algorithm. Defining the same constraint violation levels as the comparison criterion, the proposed algorithm outperforms the fixed-setup algorithm in terms of the achievable miniaturization rates, even under the optimistic scenario of having set beforehand the optimum value of $\beta$ for the latter: (a) Antenna I, (b) Antenna II, (c) Antenna III.

\section{Conclusions}

This paper proposed a novel algorithm for optimization-based antenna miniaturization using local trust region gradient search routines. Our methodology incorporates the frameworks employing a penalty function approach for handling design constraints in an implicit manner. Therein, the appropriate adjustment of the objective function, specifically the penalty terms, has a strong correlation with the reliability as well as the efficacy of the optimization process, both in terms of the achievable miniaturization rate and constraint satisfaction. The proposed methodology effectively eliminates the need for trial-and-error efforts by automated adaptive adjustment of the penalty coefficients. The latter is based on a sufficiency of constraint violation improvement between consecutive iterations throughout the entire optimization process. The optimum values of penalty coefficients are identified for particular iterations and consequently enabling the optimization process to realize a 
precise control over the constraint violations while leading to overall better results in terms of the achievable miniaturization rates as compared to the fixed adjustment setup.

The proposed procedure has been validated using three broadband antenna structures optimized for minimum size with constraints imposed on their maximum in-band reflection coefficient. Benchmarking the against the fixed penalty coefficient setups indicates superiority of automated adaptive adjustment setup in terms of achieving smaller size with a precise control over the constraint violations. The future work will involve a more comprehensive validation of the methodology in applications featuring multiple constraints, e.g., circularly polarized antennas, high-gain antennas, etc.

Author Contributions: Conceptualization, M.M. and S.K.; methodology, M.M. and S.K.; software, M.M.; validation, M.M.; formal analysis, S.K.; investigation, M.M.; resources, S.K.; data curation, M.M.; writing—original draft preparation, M.M. and S.K.; writing—review and editing, M.M. and S.K.; visualization, M.M.; supervision, S.K.; project administration, S.K.; funding acquisition, S.K. All authors have read and agreed to the published version of the manuscript.

Funding: This work was supported in part by the Icelandic Centre for Research (RANNIS) Grant 206606, and by National Science Centre of Poland Grant 2017/27/B/ST7/00563.

Acknowledgments: The authors thank Dassault Systemes, France, for making CST Microwave Studio available.

Conflicts of Interest: The authors declare no conflict of interest. The funders had no role in the design of the study; in the collection, analyses, or interpretation of data; in the writing of the manuscript, or in the decision to publish the results.

\section{References}

1. Le, T.T.; Yun, T.-Y. Miniaturization of a dual band wearable antenna for dual-band WBAN applications. IEEE Antennas Wirel. Propag. Lett. 2020, 19, 1452-1456. [CrossRef]

2. Agneessens, S.; Rogier, H. Compact half diamond dual-band textile HMSIW on-body antenna. IEEE Trans. Antennas Propag. 2014, 62, 2374-2381. [CrossRef]

3. Upadhyay, D.; Dwivedi, R.P. Antenna miniaturization techniques for wireless applications. In Proceedings of the 2014 Eleventh International Conference on Wireless and Optical Communication Networks (WOCN), Vijayawada, India, 11-13 September 2014; pp. $1-4$.

4. Oraizi, H.; Rezai, B. Dual-banding and miniaturization of planar triangular monopole antenna by inductive and dielectric loadings. IEEE Antennas Wirel. Propag. Lett. 2013, 12, 1594-1597. [CrossRef]

5. Oh, J.; Sarabandi, K. A topology-based miniaturization of circularly polarized patch antennas. IEEE Trans. Antennas Propag. 2013, 61, 1422-1426. [CrossRef]

6. Abbosh, A.M. Miniaturized microstrip-fed tapered-slot antenna with altrawideband performance. IEEE Antennas Wirel. Propag. Lett. 2009, 8, 690-692. [CrossRef]

7. Abbosh, A.M. Miniaturization of planar ultrawideband antenna via corrugation. IEEE Antennas Wirel. Propag. Lett. 2008, 7 , 685-688. [CrossRef]

8. Al-Azza, A.A.; Al-Jodah, A.A. Spider monkey optimization: A novel technique for antenna optimization. IEEE Antennas Wirel. Propag. Lett. 2016, 15, 1016-1019. [CrossRef]

9. Lalbakhsh, A.; Afzal, M.U.; Esselle, K.P. Multiobjective particle swarm optimization to design a time-delay equalizer metasurface for an electromagnetic band-gap resonator antenna. IEEE Antennas Wirel. Propag. Lett. 2017, 16, 912-915. [CrossRef]

10. Goudos, S.K.; Siakavara, K.; Samaras, T.; Vafiadis, E.E.; Sahalos, J.N. Self-adaptive differential evolution applied to real-valued antenna and microwave design problems. IEEE Trans. Antennas Propag. 2011, 59, 1286-1298. [CrossRef]

11. Tomasson, J.A.; Koziel, S.; Pietrenko Dabrowska, A. Quasi-global optimization of antenna structures using principal components and affine subspace-spanned surrogates. IEEE Access 2020, 8, 50078-50084. [CrossRef]

12. Koziel, S.; Pietrenko Dabrowska, A. Performance-based nested surrogate modeling of antenna input characteristics. IEEE Trans. Antennas Propag. 2019, 67, 2904-2912. [CrossRef]

13. Song, Y.; Cheng, Q.S.; Koziel, S. Multi-fidelity local surrogate model for computationally efficient microwave component design optimization. Sensors 2019, 19, 3023. [CrossRef]

14. Director, S.; Rohrer, R. The generalized adjoint network and network sensitivities. IEEE Trans. Circuit Theory 1969, 16, 318-323. [CrossRef]

15. Paronneau, O. Optimal Shape Design for Elliptic Systems; Springer: Berlin/Heidelberg, Germany, 1982; Volume 3, pp. 42-66.

16. Jameson, A. Aerodynamic design via control theory. J. Sci. Comput. 1988, 3, 233-260. [CrossRef]

17. El Sabbagh, M.A.; Bakr, M.H.; Nilolova, N.K. Sensitivity analysis of the scattering parameters of microwave filters using the adjoint network method. Int. J. RF Microw. Comput. Aided Eng. 2006, 16, 569-606. [CrossRef] 
18. Papadimitriou, D.; Giannakoglou, K. Aerodynamic shape optimization using first and second order adjoint and direct approaches. Arch. Comput. Methods Eng. 2008, 15, 447-488. [CrossRef]

19. Toivann, J.I.; Mäkinen, R.A.E.; Järvenpää, S.; Ylä-Oijala, P.; Rahola, J. Electromagnetic sensitivity analysis and shape optimization using method of moments and automatic differentiation. IEEE Trans. Antennas Propag. 2009, 57, 168-175. [CrossRef]

20. Koziel, S.; Ogurtsov, S. Model management for cost-efficient surrogate-based optimization of antennas using variable-fidelity electromagnetic simulations. IET Microw. Antennas Propag. 2012, 6, 1643-1650. [CrossRef]

21. Koziel, S.; Ogurtsov, S. Low-fidelity model considerations for simulation-based optimization of miniaturized wideband antennas. IET Microw. Antennas Propag. 2012, 12, 1613-1619. [CrossRef]

22. Koziel, S.; Ogurtsov, S. Robust multi-fidelity simulation-driven optimization of microwave structures. In Proceedings of the 2010 IEEE MTT-S International Microwave Symposium, Anaheim, CA, USA, 23-28 May 2010; pp. 201-204.

23. De Villiers, D.I.L.; Couckuyt, I.; Dhaene, T. Multi-objective optimization of reflector antennas using Kriging and probability of improvement. In Proceedings of the IEEE International Symposium on Antennas and Propagation \& USNC/URSI National Radio Science Meeting, San Diego, CA, USA, 9-14 July 2017; pp. 985-986.

24. Bandler, J.W.; Biernacki, R.M.; Chen, S.H.; Grobelny, P.A.; Hemmers, R.H. Space mapping technique for electromagnetic optimization. IEEE Trans. Microw. Theory Technol. 1994, 42, 2536-2544. [CrossRef]

25. Ossorio, J.; Melgarejo, J.C.; Boria, V.E.; Guglielmi, M.; Bandler, J.W. On the alignment of low-fidelity and high-fidelity simulation spaces for the design of microwave waveguide filters. IEEE Trans. Microw. Theory Technol. 2018, 66, 5183-5196. [CrossRef]

26. Bandler, J.W.; Cheng, Q.S.; Georgieva, N.; Ismail, M.A. Implicit space mapping EM-based modeling and design exploiting preassigned parameters. In Proceedings of the 2002 IEEE MTT-S International Microwave Symposium Digest (Cat. No. 02CH37278), Seattle, WA, USA, 2-7 June 2002; Volume 2, pp. 713-716.

27. Koziel, S.; Ogurtsov, S. Fast surrogate-assisted simulation-driven optimization of add-drop resonators for integrated photonic circuits. IET Microw. Ant. Propag. 2015, 9, 672-675. [CrossRef]

28. Xiao, L.; Shao, W.; Ding, X.; Wang, B. Dynamic adjustment kernel extreme learning machine for microwave component design. IEEE Trans. Microw. Theory Technol. 2018, 66, 4452-4461. [CrossRef]

29. Zhang, C.; Zhu, Y.; Cheng, Q.; Fu, H.; Ma, J.; Zhang, Q. Extreme learning machine for the behavioral modeling of RF power amplifiers. In Proceedings of the 2017 IEEE MTT-S International Microwave Symposium (IMS), Honololu, HI, USA, 4-9 June 2017; pp. 558-561.

30. Lim, D.K.; Woo, D.K.; Yeo, H.K.; Jung, S.Y.; Ro, J.S.; Jung, H.K. A novel surrogate-assisted multi-objective optimization algorithm for an electromagnetic machine design. IEEE Trans. Magn. 2015, 51, 1-4.

31. Toktas, A.; Ustun, D.; Tekbas, M. Multi-objective design of multi-layer radar absorber using surrogate-based optimization. IEEE Trans. Microw. Theory Technol. 2019, 67, 3318-3329. [CrossRef]

32. Koziel, S.; Pietrenko Dabrowska, A. Reduced-cost design closure of antennas by means of gradient search with restricted sensitivity update. Metrol. Meas. Syst. 2019, 26, 595-605.

33. Haq, M.A.; Koziel, S. Ground plane alterations for design of high-isolation compact wideband MIMO antenna. IEEE Access 2018, 6, 48978-48983. [CrossRef]

34. Johanesson, D.O.; Koziel, S. Feasible space boundary search for improved optimization-based miniaturization of antenna structures. Microw. Antennas Propag. 2018, 12, 1273-1278. [CrossRef]

35. Johanesson, D.O.; Koziel, S.; Bekasiewicz, A. EM-driven constrained miniaturization of antennas using adaptive in-band reflection acceptance threshold. Int. J. Numer. Model. Electron. Netw. Devices Fields 2018, 32, e2513. [CrossRef]

36. Alsath, M.G.N.; Kanagasabai, M. Compact UWB monopole antenna for automotive communications. IEEE Trans. Antennas Propag. 2015, 63, 4204-4208. [CrossRef]

37. Haq, M.A.; Koziel, S. Simulation-based optimization for rigorous assessment of ground plane modifications in compact UWB antenna design. Int. J. RF Microw. Comput. Aided Eng. 2018, 28, e21204. [CrossRef]

38. Koziel, S.; Bekasiewicz, A. Comprehensive comparison of compact UWB antenna performance by means of multi-objective optimization. IEEE Trans. Antennas Propag. 2017, 65, 3427-3436. [CrossRef] 